\title{
IBD specialists identify ten top priorities for comparative effectiveness research
}

A

group of IBD specialists is

embracing the field of comparative effectiveness research (CER) and has developed a list of ten topics that they suggest could be top priorities for future IBD research.

The Institute of Medicine (IOM) defines CER as "the generation and synthesis of evidence that compares the benefits and harms of alternative methods to prevent, diagnose, treat, and monitor a clinical condition or to improve the delivery of care." Health-care decisions can sometimes be a struggle for clinicians, owing to a lack of reliable evidence. Randomized, controlled trials are usually considered the gold standard of evidence for a particular intervention, but even these have their shortcomings. For example, most reports in the medical literature focus on placebocontrolled trials, which do not accurately reflect a typical scenario in clinical practice. Thus, some of the key components of CER are to directly compare effective interventions and to evaluate the application of these interventions in day-to-day clinical practice.

"CER is an emerging field that will undoubtedly affect the landscape of research," explains Adam Cheifetz, corresponding author of the study and Director of the Center for Inflammatory Bowel Disease, Beth Israel Deaconess Medical Center, Boston, MA. In the USA, in particular, CER is proving to be more and more important as it is expected and hoped to help in the push towards increased value from the health-care system. In June 2009, the IOM published a report of the top 100 national priorities for CER (www.iom.edu/cerpriorities).

"To date, the IOM method has not been used to develop CER priorities for a specific disease or specialty, and the feasibility of this process outside of a large government-funded effort has also not been assessed," says Cheifetz. "Therefore, we chose to adapt the methods developed by the IOM to determine feasibility and

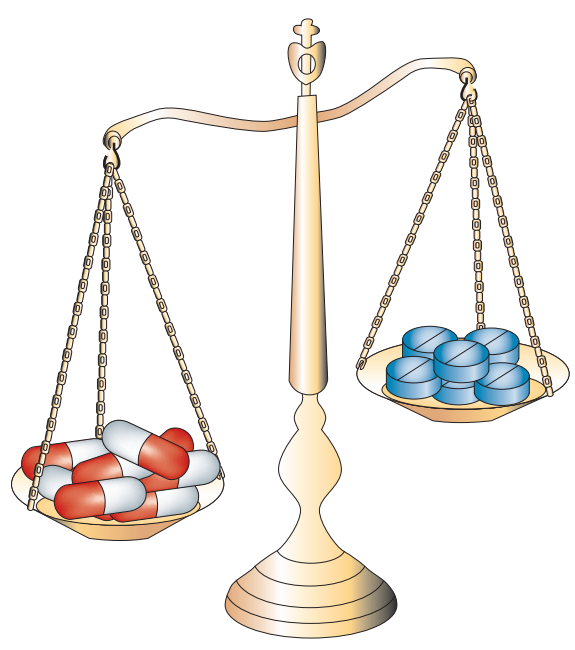

establish a list of high-priority CER topics specifically related to the care of patients with IBD."

The first step in the process was to gather input about potential CER topics from an array of 'stakeholders', including both physicians and patients. Thus, Cheifetz et al. conducted a survey of practicing gastroenterologists and asked them to suggest what they felt were important CER topics. 245 physicians (almost all from the USA, Canada, the UK and Australia) responded to the survey. Members of the 13-strong, international BRIDGe (Building Research in Inflammatory Bowel Disease Globally) group, who conducted the study, were also asked to submit their ideas. In addition, two patient focus groups were formed and each produced a list of CER topics.

The next step was that of prioritization. All in all, 341 candidate IBD CER topics were suggested. Some of these topics could be discarded owing to a lack of face validity (for example, inconsistency with the IOM definition of CER or topic considered too vague) or duplication. The remaining CER topics were then presented to the BRIDGe group, who rated, discussed and voted on topics, which finally lead to the generation of the top ten list.
The final top ten IBD CER topics were related to: biomarkers (for predicting prognosis and response to therapy); withdrawal of anti-TNF therapy or immunomodulators for patients with Crohn's disease in remission; mucosal healing as an end point of treatment; standard infliximab dosing versus trough-level dosing; anti-TNF monotherapy versus combination therapy, particularly in patients who don't respond to thiopurines; safety of long-term treatment options; anti-TNF therapy versus thiopurines for prevention of postoperative recurrence; and treatment options for steroid-refractory ulcerative colitis.

The authors emphasize that the identification of these topics could be useful for researchers and funding agencies deciding on future IBD research priorities. "We were able to show that the methodology we used is feasible and could also be utilized by other groups interested in developing a priority list of CER in their particular specialties," concludes Cheifetz. Indeed, Hal F. Yee, Chief Medical Officer, Los Angeles County Department of Health Services, who is interested in CER, comments that he "expects that insurance companies and large accountable care organizations in the USA will be pursuing these kinds of approaches as financing moves away from a fee-for-service model".

However, the authors also suggest that the prioritization of topics should be an ongoing process. The IBD knowledge base is constantly expanding, so continuous re-evaluation will be necessary, hopefully even more so as the effect of CER kicks in and questions are answered.

Isobel Franks

Original article Cheifetz, A. S. et al. Setting priorities for comparative effectiveness research in inflammatory bowel disease: results of an international provider study, expert RAND panel, and patient focus group. Inflamm. Bowel Dis. doi:10.1002/ibd.22920 